University of New Hampshire

University of New Hampshire Scholars' Repository

Physics Scholarship

Physics

7-6-2011

\title{
Neutral atom imaging of the magnetospheric cusps
}

S. M. Petrinec

M. A. Dayeh

H. O. Funsten

S. A. Fuselier

David F. Heirtzler

University of New Hampshire, David.Heirtzler@unh.edu

See next page for additional authors

Follow this and additional works at: https://scholars.unh.edu/physics_facpub

Part of the Physics Commons

\section{Recommended Citation}

Petrinec, S. M.; Dayeh, M. A.; Funsten, H. O.; Fuselier, S. A.; Heirtzler, David F.; Janzen, P.; Kucharek, Harald A.; McComas, D. J.; Moebius, Eberhard; Moore, T. E.; Reisenfeld, D. B.; Schwadron, Nathan A.; Trattner, K. J.; and Wurz, P., "Neutral atom imaging of the magnetospheric cusps" (2011). Journal of Geophysical Research-Space Physics. 78.

https://scholars.unh.edu/physics_facpub/78

This Article is brought to you for free and open access by the Physics at University of New Hampshire Scholars' Repository. It has been accepted for inclusion in Physics Scholarship by an authorized administrator of University of New Hampshire Scholars' Repository. For more information, please contact Scholarly.Communication@unh.edu. 


\section{Authors}

S. M. Petrinec, M. A. Dayeh, H. O. Funsten, S. A. Fuselier, David F. Heirtzler, P. Janzen, Harald A. Kucharek, D. J. McComas, Eberhard Moebius, T. E. Moore, D. B. Reisenfeld, Nathan A. Schwadron, K. J. Trattner, and P. Wurz 


\title{
Neutral atom imaging of the magnetospheric cusps
}

\author{
S. M. Petrinec, ${ }^{1}$ M. A. Dayeh, ${ }^{2}$ H. O. Funsten, ${ }^{3}$ S. A. Fuselier, ${ }^{1}$ D. Heirtzler, ${ }^{4}$ P. Janzen, ${ }^{5}$ \\ H. Kucharek, ${ }^{4}$ D. J. McComas, ${ }^{2,6}$ E. Möbius, ${ }^{4}$ T. E. Moore, ${ }^{7}$ D. B. Reisenfeld, ${ }^{5}$ \\ N. A. Schwadron, ${ }^{8}$ K. J. Trattner, ${ }^{1}$ and P. Wurz ${ }^{9}$
}

Received 7 December 2010; revised 22 February 2011; accepted 1 April 2011; published 6 July 2011.

[1] The magnetospheric cusps separate closed dayside magnetospheric field lines from open field lines of the magnetotail mantle and lobes. All magnetospheric field lines that map to the magnetopause also pass through the cusp regions. Thus whenever magnetic reconnection occurs at the magnetopause, magnetosheath plasma can enter one or both of the cusp regions and charge exchange with the geocorona. The resulting energetic neutral atoms (ENAs) resulting from this charge exchange process propagate away from the cusps and are observed remotely by the Interstellar Boundary Explorer (IBEX). The asymmetry of the ENA intensities between the northern and southern cusps are strongly dependent upon the Earth's dipole tilt angle and are consistent with in situ cusp observations. These asymmetric fluxes in the cusp regions are suggested to be explained by the regions at the magnetopause where magnetic reconnection is expected.

Citation: Petrinec, S. M., et al. (2011), Neutral atom imaging of the magnetospheric cusps, J. Geophys. Res., 116, A07203, doi:10.1029/2010JA016357.

\section{Introduction}

[2] The magnetospheric cusps have long been regions of considerable interest [e.g., Shelley et al., 1976; Paschmann et al., 1976; Haerendel et al., 1978]. The cusp regions are filled with plasma over a broad range of energies and have been sampled by various satellite instruments over a considerable range of altitudes. The cusp location, size, and orientation, as well as cusp plasma properties (e.g., composition and fluxes versus energy/time/latitude; wave/ particle interactions) depend on the season and the solar wind conditions. Thus while in situ observations can be quite detailed [e.g., Dubinin et al., 2002; Nykyri et al., 2003; Cargill et al., 2004; Trattner et al., 2005, 2008, and references therein], it is often difficult to discriminate between temporal and spatial effects within the cusp. Now, with the launch of the IBEX satellite (see McComas et al. [2009a] and other papers in the IBEX special issue of Space Science

\footnotetext{
${ }^{1}$ Lockheed Martin Advanced Technology Center, Palo Alto, California, USA.

${ }^{2}$ Southwest Research Institute, San Antonio, Texas, USA.

${ }^{3}$ Los Alamos National Laboratory, Los Alamos, New Mexico, USA.

${ }^{4}$ Institute for the Study of Earth, Oceans and Space, University of New Hampshire, Durham, New Hampshire, USA.

${ }^{5}$ Department of Physics and Astronomy, University of Montana, Billings, Montana, USA.

${ }^{6}$ Space Science and Engineering Division, University of Texas, San Antonio, Texas, USA.

${ }^{7}$ Heliophysics Science Division, NASA Goddard Space Flight Center, Greenbelt, Maryland, USA.

${ }^{8}$ Astronomy Department, Boston University, Boston, Massachusetts, USA.

${ }^{9}$ Physics Institute, University of Bern, Bern, Switzerland.

Copyright 2011 by the American Geophysical Union. 0148-0227/11/2010JA016357
}

Reviews), it is possible to remotely image the cusp regions, using the charge exchange process between the cusp plasma population and the geocorona to produce energetic neutral atoms (ENAs). ENA imaging is a new and powerful method for viewing the cusps and can be used in conjunction with in situ observations to further our understanding of the interaction between the solar wind and the magnetosphere. In this study we describe the first remote observations of the cusp and examine their response as a function to varying solar wind conditions.

\section{Observations}

[3] The IBEX spacecraft is in a highly eccentric orbit about the Earth. Its spin axis is roughly Sun-pointed and the spacecraft rotates at $\sim 4$ RPM. At the start of each $\sim 7.5$ day orbit, near perigee, the spin axis is pointed slightly west of the Sun $\left(\sim 1.5^{\circ}\right)$. Over an orbit, its inertially fixed spin axis appears to drift eastward across the Sun finishing $\sim 6^{\circ}$ east of the Sun by the next perigee, which is followed by another repointing maneuver. The IBEX-Hi and -Lo sensors [Funsten et al., 2009; Fuselier et al., 2009] view perpendicular to the spin axis, collecting ENAs as a function of spin phase (measured as an angle from the north ecliptic pole or NEP). This configuration provides extremely high sensitivity ENA observations of each $\sim 7^{\circ}$ wide swath of the sky every 6 months [McComas et al., 2009a].

[4] While the primary objective of the IBEX mission is to understand the interaction region between the heliosphere and the local interstellar medium through study of ENA emission (see McComas et al. [2009b] and other papers in the special issue of Science), the instruments are of such high sensitivity as to measure other extremely dim sources of ENA emission. ENAs created by backscatter and neutralization 

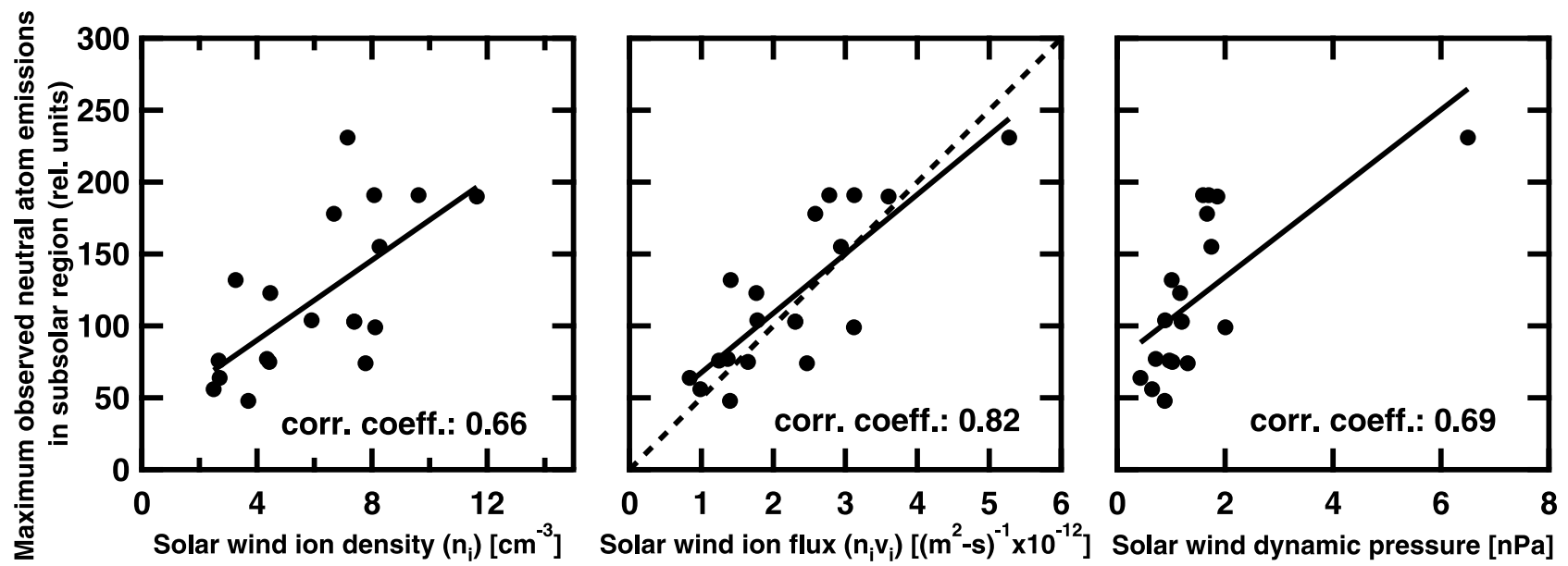

Figure 1. Correlations between the magnitude of ENA emission in the subsolar magnetopause region versus convected solar wind parameters, from 19 separate IBEX orbits. The best linear correlation is with the ion number flux, and the $y$-intercept is closest to zero.

of the solar wind by the lunar regolith were discovered using the IBEX observations [McComas et al., 2009c]. Furthermore, because IBEX imagers view approximately perpendicular to the Sun-Earth vector and the IBEX orbit is highly elliptical and near the equatorial plane, the IBEX data set is rich in views of magnetospheric structures. The ENA observations of the magnetospheric regions are necessarily line-of-sight integrations [Wurz, 2000]. When the line-ofsight emission is projected into the noon-midnight meridian plane of the magnetosphere, the IBEX instrument field of view covers between $\sim 3.7 \mathrm{R}_{\mathrm{E}}$ (when IBEX is just outside the bow shock) and $\sim 6.1 \mathrm{R}_{\mathrm{E}}$ (when IBEX is at apogee). This is the inherent spatial resolution of the instruments. Assuming that the plasma interaction in the region of interest does not change appreciably with time, the slowly changing view of the magnetosphere allows us to build up a composite image over many spacecraft spins. This is described in greater detail in the following section. The subsolar location of the magnetopause is one source of ENA emission, as observed recently by Fuselier et al. [2010]. Additionally, ENA emission has been observed remotely from the plasma sheet by IBEX, revealing time variation indicative of dynamic processes in this region [McComas et al., 2011]. Here, we focus on ENA emission from the magnetospheric cusps.

\section{Results}

[5] In the study of Fuselier et al. [2010], ENA emission is observed in the subsolar magnetopause region, and the magnitude of this emission is observed to vary from orbit to orbit. To determine what parameter of the solar wind best orders the variation of the subsolar magnetopause emissions, we extend the Fuselier et al. [2010] study by comparing the peak ENA intensities from the composite images from each of 18 separate orbits with the mean values of solar wind ion density $n_{i}$, number flux $n_{i} v_{i}$, and dynamic pressure $m_{i} n_{i} v_{i}^{2}$ (Figure 1) as measured by the Wind spacecraft. The "subsolar region" is somewhat loosely defined; the region was selected from each composite image by hand, while trying to exclude emission from other regions. The selected regions about the subsolar point were a few $R_{E}$ in extent in both the $\mathrm{X}_{\mathrm{GSE}}$ and $\mathrm{Z}_{\mathrm{GSE}}$ directions. Throughout this study we have selected energy step 3 (0.9-1.5 keV FWHM) of the IBEX-Hi instrument because it is most representative of both the bulk solar wind and magnetosheath plasma energy. In comparing the ENA emission with the solar wind properties, a simple linear regression analysis reveals that the ion number flux correlates best with the ENA intensity, i.e., the correlation coefficient is highest and the $y$ intercept is near zero (see Figure 1). This result is particularly useful for normalizing cusp emissions, which are thought to result from magnetosheath plasma that has undergone magnetic reconnection, entered the cusps, and charge exchanged with the geocorona. The cusps consist of plasma with energy/time dispersion signatures supportive of magnetopause magnetic reconnection, whether the interplanetary magnetic field (IMF) is directed southward or northward [e.g., Newell and Meng, 1991; Escoubet et al., 1992; Lockwood and Smith, 1992, 1994; Topliss et al., 2000; Trattner et al., 2002a, 2002b].

[6] To illustrate the effect of normalization of the cusp emissions, a few representative IBEX orbits have been chosen (Figure 2). The cusp ENA observations for three different orbits are ordered here according to solar wind ion number flux. The top row shows the solar wind number flux over the time of the ENA observations in the cusp. The solar wind data is time-adjusted based on the speed to compensate for the travel time from the Wind location to the cusps. The middle row shows the measured ENA counts of energy

Figure 2. $(\mathrm{a}-\mathrm{c})$ Solar wind number flux (low, medium, and high) as measured by the Wind spacecraft, for three different IBEX viewing intervals of the cusp regions. (d-f) Composite images of the dayside magnetosphere using the ENA observations (IBEX-Hi in the energy range $0.9-1.5 \mathrm{keV}$ ), as projected into the $\mathrm{Y}_{\mathrm{GSM}}=0$ plane. (g-i) The same composite images, but each time step (i.e., vertical sweep) has been normalized by the convected solar wind number flux. 



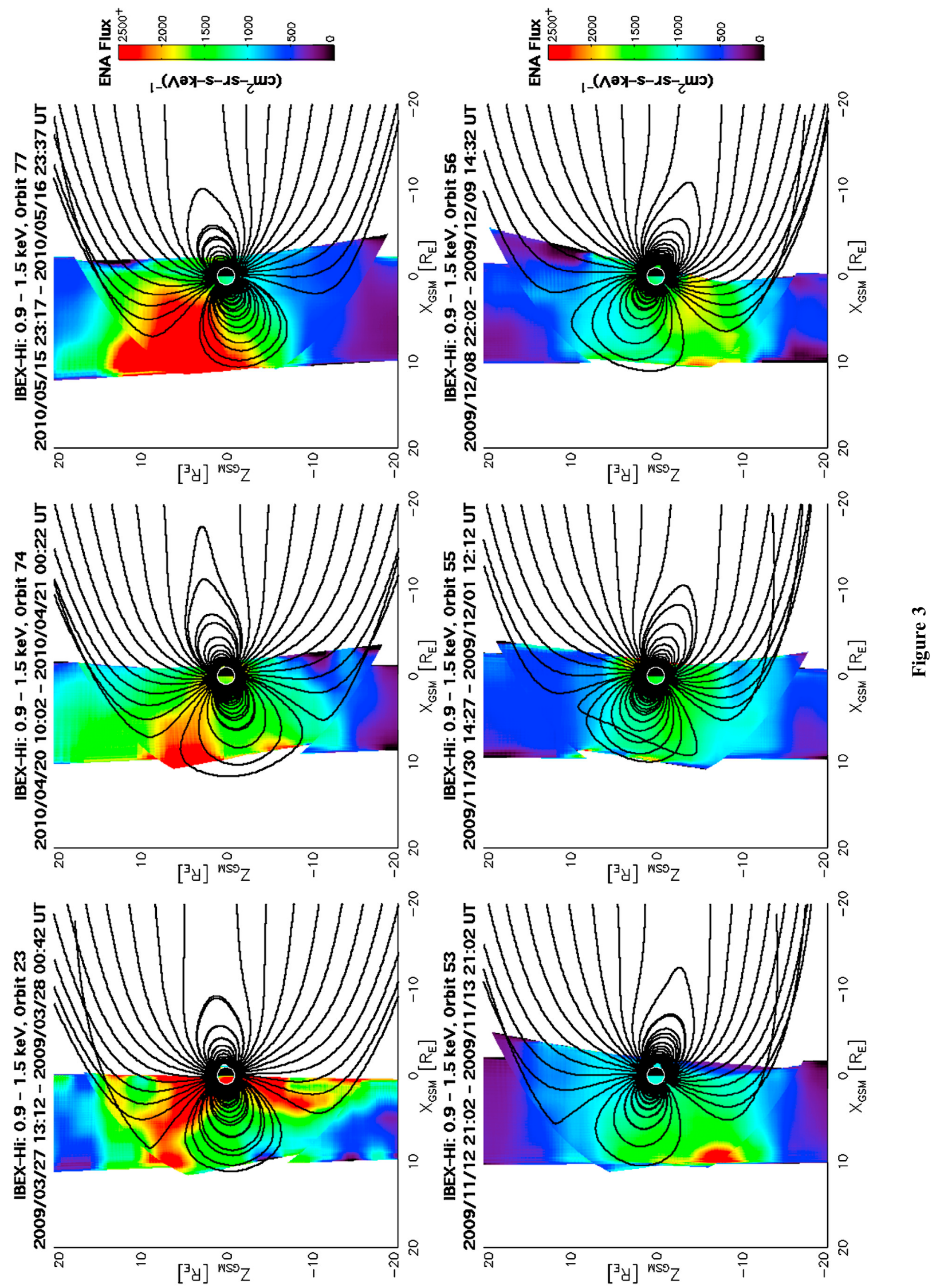

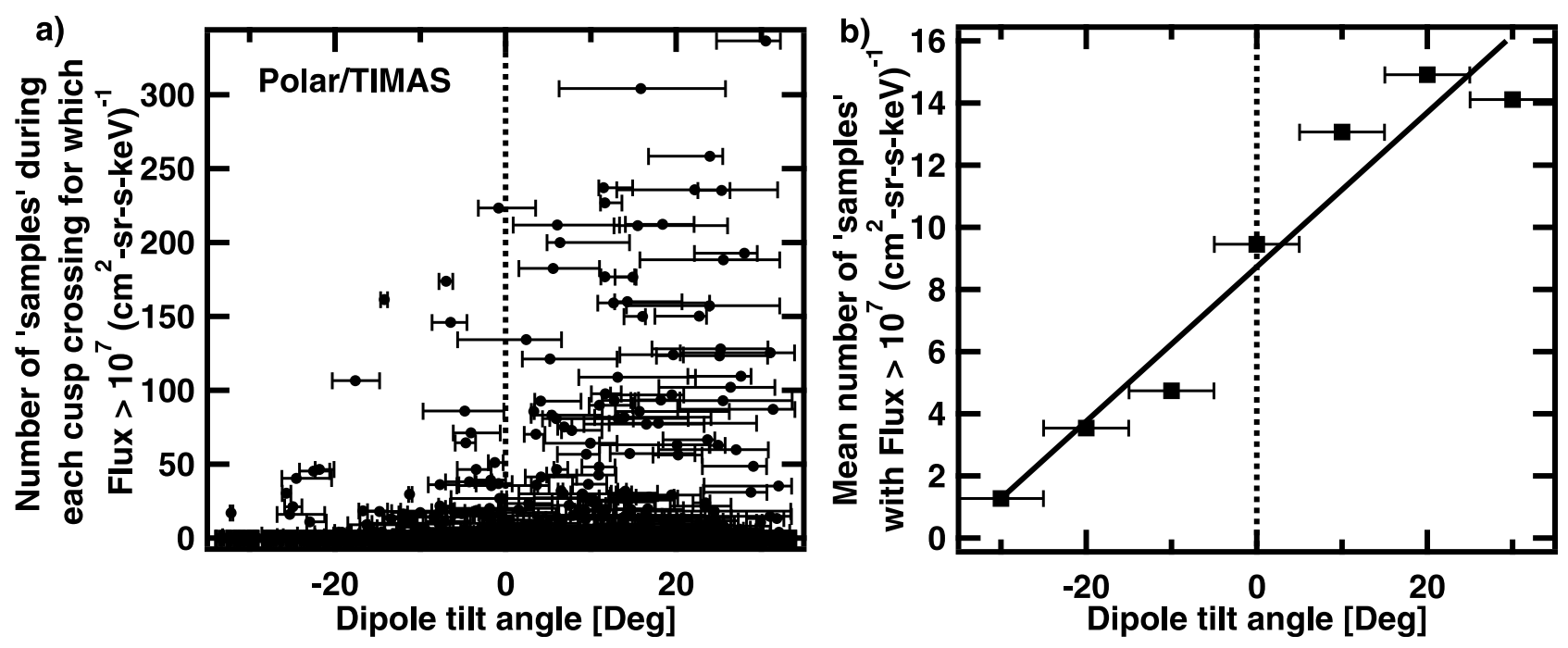

Figure 4. (a) Time intervals during each Polar pass through the northern cusp for which the number flux at $E=1 \mathrm{keV}$ is $>10^{7}\left(\mathrm{~cm}^{2} \mathrm{sr} \mathrm{s} \mathrm{keV}\right)^{-1}$. (b) The mean number of time intervals within each $10^{\circ}$ dipole tilt angle bin.

step 3 for the corresponding time intervals. Images are built up from many spins of the IBEX spacecraft. As the IBEX spacecraft progresses through its orbit, and as its spin direction slowly precesses, the view of the magnetosphere slowly changes. Because of the movement of the IBEX spacecraft relative to Earth, the vertical swaths mapped out from one spin to the next almost, but not quite, exactly overlap. Overlapped regions are averaged; thus the final image has the appearance of having greater spatial resolution than the inherent resolution provided by the $\sim 7^{\circ}$ (FWHM) conical field of view.

[7] Superimposed on the ENA images are magnetospheric field lines in the noon-midnight meridian plane from the Tsyganenko [1995] magnetic field model (based on Tsyganenko [1995] with minor modifications to some routines). The dipole tilt angle used represents the median tilt angle during the interval of interest. Although the gross field structure is quite different for each of the three intervals, elevated ENA emission clearly appears in the cusp regions of the magnetic field model. Therefore, although ENA observations are lineof-sight (LOS) integrations over long distances and do not provide information on the distance to the cusp source, we reasonably assume that the ENA emission source is where the LOS passes closest to the Earth (i.e., close to the GSM noonmeridian plane).

[8] Figures $2 \mathrm{~g}-2 \mathrm{i}$ are the same as Figures $2 \mathrm{~d}-2 \mathrm{f}$, except that with each spin of the IBEX spacecraft (and corresponding vertical swath of observations), the ENA flux is normalized by the convected solar wind number flux. Comparing Figures $2 g-2 i$ with Figures $2 d-2 f$, it is evident that normalization reduces the overall variability between the different orbits. In Figures $2 \mathrm{a}, 2 \mathrm{~d}$, and $2 \mathrm{~g}$ and Figures $2 \mathrm{c}$, $2 \mathrm{f}$, and $2 \mathrm{i}$, it is also evident that for intervals of positive dipole tilt angle (northern summer), the northern cusp exhibits a greater ENA flux than does the southern cusp. The opposite occurs for negative dipole tilt angles (northern winter, Figures $2 \mathrm{~b}, 2 \mathrm{e}$, and $2 \mathrm{~h}$ ).

[9] Figure 3 shows the cusp ENA emissions from several of the IBEX orbits; where the ENA emission for each spin is normalized to the convected solar wind number flux corresponding to that specific time. In addition, within the magnetopause from the Tsyganenko model the projected (into the $\mathrm{Y}_{\mathrm{GSM}}=0$ noon-meridian plane) emission locations are adjusted so as to maintain the relative location with respect to the cusp and to the magnetopause. This adjustment is analogous to that performed by Lavraud et al. [2005, Figures $1 \mathrm{~b}$ and $1 \mathrm{c}]$ in their statistical examination of cusp region observations from the Cluster mission for different times and dipole tilt angles. As in Figure 2, in the intervals of ENA emission shown in Figure 3 the relative brightness of the northern or southern cusp regions is closely associated with the tilt of the magnetosphere; specifically, the cusp region oriented toward the Sun is the one with the greatest ENA emissions. The one exception is orbit 23, which occurred close to the vernal equinox and for which northern and southern cusp emissions are of strong, nearly equal intensity.

[10] In situ measurements of the source ion population in the northern and southern cusp regions show a similar seasonal asymmetry as the observed ENA emissions. Observations of proton flux within the northern cusp region by the Toroidal Imaging Mass-Angle Spectrograph (TIMAS) on board the Polar spacecraft shows similar behavior to the present cusp ENA observations. For 1094 cusp crossings over 3 years, the number of time intervals for which the differential number flux at $E=1 \mathrm{keV}$ was $>10^{7}\left(\mathrm{~cm}^{2} \mathrm{sr} \mathrm{s} \mathrm{keV}\right)^{-1}$ have been accumulated as a function of dipole tilt angle (Figure 4a). A greater number of large ion flux intervals is observed for

Figure 3. Several cusp observation intervals, as detected with ENAs. Despite normalization of ENA count rate to solar wind ion flux, some ENA flux differences from one orbit to another remain due to the influence of other parameters (e.g., the average cusp emission flux is $\sim 25 \%$ larger for southward IMF than for northward IMF). 

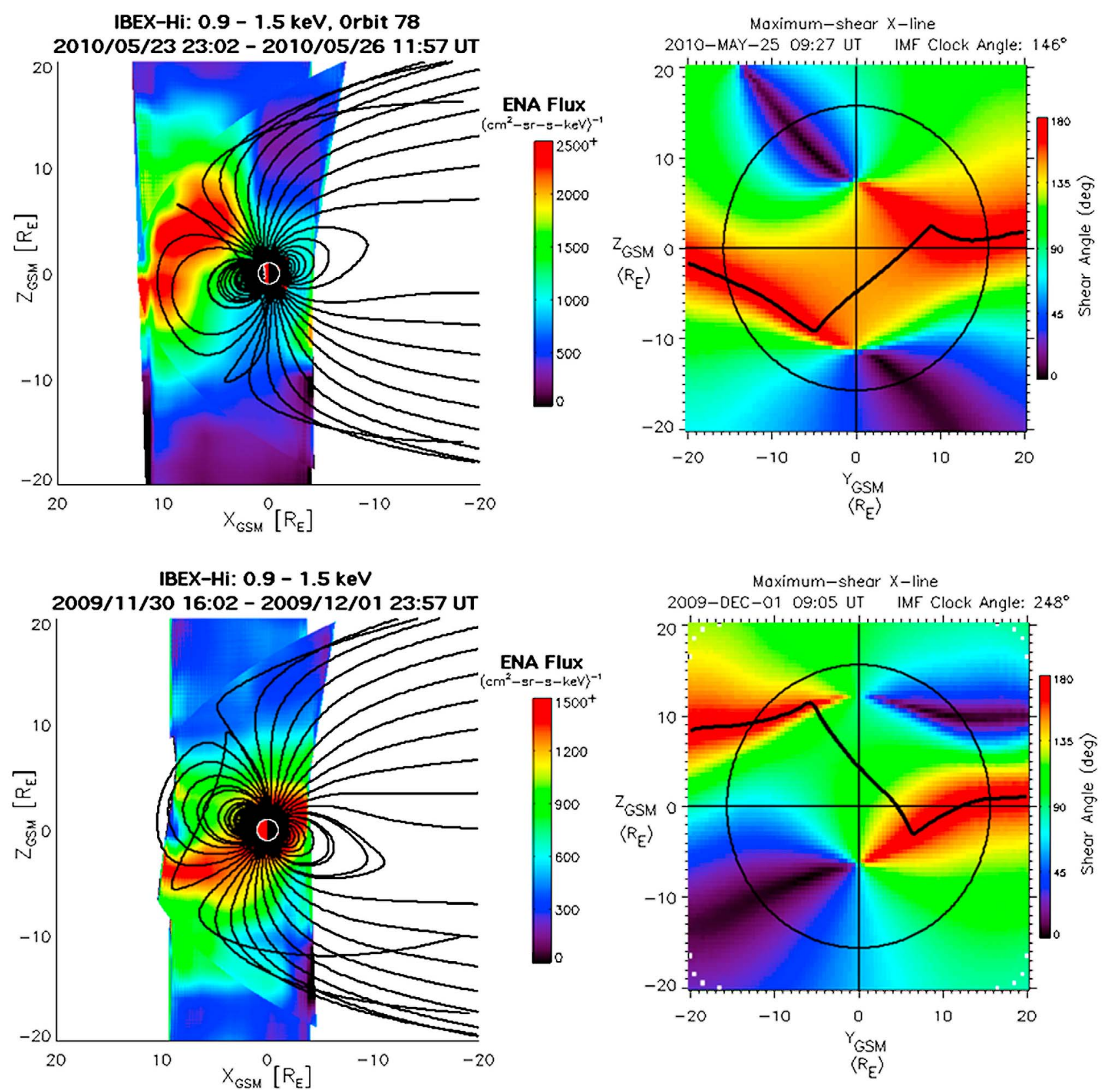

Figure 5. (left) Two ENA composite images, for different IBEX orbits. During these time intervals, the IMF was predominantly southward, but of opposite IMF $B_{y}$. (right) The maximum magnetic shear $X$ line reconnection model for these intervals.

large positive dipole tilt angles than for negative tilt angles (median values are shown in Figure $4 b$ ). Note that this analysis is rather crude, since it does not properly account for a potential orbital bias of the Polar spacecraft as a function of season. Additional support for the asymmetry of the source ion fluxes in the north and south cusp regions comes from a recent study by Newell et al. [2010], using 11 years of Defense Meteorological Satellite Program (DMSP) in situ observations to produce, among other things, polar region synoptic maps \{MLT, Mlat\} of energy flux and ion number flux [Newell et al., 2010, Figures 7 and 8]. The DMSP ion number flux in the northern or southern cusp region is markedly enhanced during its summer season as compared to its winter season, where a winter/summer ratio of ion flux of 0.75 is found for high solar wind driving conditions. This compares well with the ratio we find from IBEX ENA cusp observations of approximately 0.63 ; determined by comparing midaltitude to highaltitude region emissions observed simultaneously in the two cusps for eight orbits occurring during large dipole tilt angle.

\section{Discussion}

[11] It has been shown that the proton number flux is greater in the summer cusp than in the winter cusp. In this section we explore the physical process(es) responsible for this phenomenon. Because the plasma in the cusp regions 


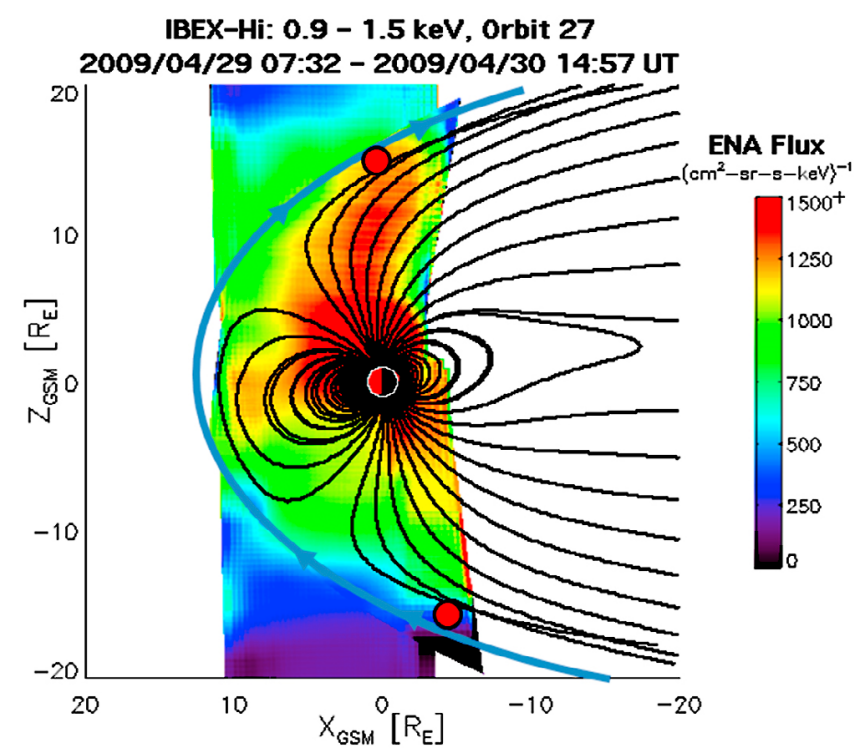

Figure 6. An ENA composite image of the cusps, during northward IMF conditions. Overlaid is the draped magnetosheath magnetic field (blue), marking the locations just tailward of the cusps where magnetic reconnection is likely to occur (red circles).

has undergone magnetic reconnection at the magnetopause and because magnetic reconnection is strongly associated with IMF orientation, IBEX ENA observations of the cusp are studied here as a function of IMF configuration, separating times of southward and northward IMF.

[12] The left column of Figure 5 shows ENA emissions from two cusp observing intervals, selected to only include times for which the IMF was southward. These intervals also had opposite senses of IMF $B_{y}$ and occurred during different seasons. As shown earlier, the summer hemisphere cusp is significantly brighter in ENA emissions than the winter hemisphere. The right column of Figure 5 shows the magnetic shear angle across the magnetopause for the midpoint of the time interval and average solar wind (southward) IMF conditions. The magnetic shear angles are determined using the Kobel and Flückiger [1994] model for the magnetosheath magnetic field, and the Tsyganenko magnetic field model just inside of the magnetopause. The thick black line illustrates where magnetic reconnection is expected to occur across the dayside magnetopause, using the maximum magnetic shear model described by Trattner et al. [2007]. It is readily seen that for positive dipole tilt angle, most of the reconnection $X$ line lies below the magnetic equator. Flux tubes reconnecting at this $X$ line and attached to the southern cusp quickly convect to the nightside, aided by both the ambient magnetosheath flow and the $\mathbf{J} \times \mathbf{B}$ force of the reconnected flux tube. Magnetosheath plasma on these field lines enters the southern cusp at higher energies, but because of rapid convection of the flux tube, the magnetosheath number flux will be low. In contrast, flux tubes reconnecting at this $X$ line and attached to the northern cusp will slowly convect toward the nightside, since reconnected flux tubes must first move against the ambient magnetosheath flow, until reaching the geomagnetic equator. As a result, magnetosheath plasma enters the cusps at lower energies than those in the southern cusp and would not be observed in this energy band. However, because the convection toward the north is slow, there is more time to allow a much greater magnetosheath number flux to enter the northern cusp and interact with the geocorona. The result of this extended interaction is that a larger number flux is observed in the northern cusp than the southern cusp for southern IMF and positive dipole tilt angle. The southern cusp appears brighter in ENAs than the northern cusp when the dipole tilt angle is negative. The situation for negative dipole tilt angle is shown in Figure 5d.

[13] An example of ENA emissions occurring during predominantly northward IMF is shown in Figure 6. Here, only northward IMF segments of the time interval have been used to create the composite image. When the IMF is northward and the dipole tilt angle is positive, reconnection occurs tailward of the cusps. It is hypothesized that for northward IMF most of the reconnection region lies tailward of, but close to, the cusps. As a result, the summer hemisphere reconnection site would lie sunward of the winter hemisphere site. This is important because the ambient magnetosheath plasma accelerates as it moves tailward. Thus the summer hemisphere reconnection site would allow more of the magnetosheath plasma distribution which has undergone reconnection to reach the cusp than would occur in the winter cusp. In fact, the winter hemisphere reconnection site may not be stable at all, if the magnetosheath Alfvén Mach number is too high (depending upon the properties of the plasma depletion layer). This scenario assumes that the IMF $B_{x}$ component is not the major component [cf. Fairfield and Scudder, 1985]; a large IMF $B_{x}$ component may complicate the reconnection topology. The result of this interaction is that, in general, a larger flux of ENAs should be observed in the summer than the winter cusp for northward IMF.

[14] The above scenarios illustrate that independent of whether the IMF is southward or northward, the cusp which is most sunward should contain the larger number flux of magnetosheath plasma which has undergone magnetic reconnection and produce more enhanced ENA emissions. The implication of this analysis is that when the IMF is southward, the dayside magnetopause reconnection $X$ line is typically not equally divided across the magnetic equator (as described by most $X$ line models), but its location is strongly influenced by the dipole tilt angle instead, as described by the Maximum Magnetic Shear model [Trattner et al., 2007]. This interpretation also explains the seasonal asymmetry in the Polar/TIMAS observations and the DMSP number flux as described by Newell et al. [2010].

[15] Acknowledgments. Solar wind data are from the Wind spacecraft and provided through the NSSDC CDA Web site. Support for this study comes from the IBEX mission as a part of NASA's Explorer program. IBEX is the result of efforts from a large number of scientists, engineers, and others; all who contributed to this mission share in its success.

[16] Masaki Fujimoto thanks the reviewers for their assistance in evaluating this paper.

\section{References}

Cargill, P. J., M. W. Dunlop, B. Lavraud, R. C. Elphic, D. L. Holland, K. Nykyri, A. Balogh, I. Dandouras, and H. Rème (2004), CLUSTER encounters with the high altitude cusp: Boundary structure and magnetic field depletions, Ann. Geophys., 22(5), 1739-1754, doi:10.5194/angeo22-1739-2004. 
Dubinin, E., et al. (2002), Polar-Interball coordinated observations of plasma and magnetic field characteristics in the regions of the northern and southern distant cusps, J. Geophys. Res., 107(A5), 1053, doi:10.1029/ 2001JA900068.

Escoubet, C. P., et al. (1992), Staircase ion signature in the polar cusp: A case study, Geophys. Res. Lett., 19, 1735-1738, doi:10.1029/92GL01806.

Fairfield, D., and J. Scudder (1985), Polar rain: Solar coronal electrons in the Earth's magnetosphere, J. Geophys. Res., 90, 4055-4068, doi:10.1029/ JA090iA05p04055.

Funsten, H. O., et al. (2009), The Interstellar Boundary Explorer High Energy (IBEX-Hi) Neutral Atom Imager, Space Sci. Rev., 146, 75-103, doi:10.1007/s11214-009-9504-y.

Fuselier, S. A., et al. (2009), The IBEX-Lo Sensor, Space Sci. Rev., 146, 117-147, doi:10.1007/s11214-009-9495-8.

Fuselier, S. A., et al. (2010), Energetic neutral atoms from the Earth's subsolar magnetopause, Geophys. Res. Lett., 37, L13101, doi:10.1029/ 2010GL044140.

Haerendel, G., G. Paschmann, N. Sckopke, H. Rosenbauer, and P. C. Hedgecock (1978), The frontside boundary layer of the magnetosphere and the problem of reconnection, J. Geophys. Res., 83, 3195-3216, doi:10.1029/JA083iA07p03195.

Kobel, E., and E. O. Flückiger (1994), A model of the steady state magnetic field in the magnetosheath, J. Geophys. Res., 99, 23,617-23,622, doi:10.1029/94JA01778.

Lavraud, B., A. Fedorov, E. Budnik, M. F. Thomsen, A. Grigoriev, P. J. Cargill, M. W. Dunlop, H. Réme, I. Dandouras, and A. Balogh (2005), High-altitude cusp flow dependence on IMF orientation: A 3-year Cluster statistical study, J. Geophys. Res., 110, A02209, doi:10.1029/2004JA010804.

Lockwood, M., and M. F. Smith (1992), The variation of reconnection rate at the dayside magnetopause and cusp ion precipitation, J. Geophys. Res. 97, 14,841-14,847, doi:10.1029/92JA01261.

Lockwood, M., and M. F. Smith (1994), Low- and mid-altitude cusp particle signatures for general magnetopause reconnection rate variations, 1, Theory, J. Geophys. Res., 99, 8531-8553, doi:10.1029/93JA03399.

McComas, D. J., et al. (2009a), IBEX-Interstellar Boundary Explorer, Space Sci. Rev., 146, 11-33, doi:10.1007/s11214-009-9499-4.

McComas, D. J., et al. (2009b), Global observations of the interstellar interaction from the Interstellar Boundary Explorer (IBEX), Science, 326 959-962, doi:10.1126/science. 1180906 .

McComas, D. J., et al. (2009c), Lunar backscatter and neutralization of the solar wind: First observations of neutral atoms from the Moon, Geophys. Res. Lett., 36, L12104, doi:10.1029/2009GL038794.

McComas, D. J., M. A. Dayeh, H. O. Funsten, S. A. Fuselier, J. Goldstein, J.-M. Jahn, P. Janzen, S. M. Petrinec, D. B. Reisenfeld, and N. A Schwadron (2011), First IBEX observations of the terrestrial plasma sheet and a possible disconnection event, J. Geophys. Res., 116, A02211, doi:10.1029/2010JA016138.

Newell, P. T., and C.-I. Meng (1991), Ion acceleration at the equatorward edge of the cusp: Low altitude observations of patchy merging, Geophys. Res. Lett., 18, 1829-1832, doi:10.1029/91GL02088.

Newell, P. T., T. Sotirelis, and S. Wing (2010), Seasonal variations in diffuse, monoenergetic, and broadband aurora, J. Geophys. Res., 115, A03216, doi:10.1029/2009JA014805.

Nykyri, K., P. J. Cargill, E. A. Lucek, T. S. Horbury, A. Balogh, B. Lavraud, I. Dandouras, and H. Rème (2003), Ion cyclotron waves in the high altitude cusp: CLUSTER observations at varying spacecraft separations, Geophys. Res. Lett., 30(24), 2263, doi:10.1029/2003GL018594.

Paschmann, G., G. Haerendel, N. Sckopke, H. Rosenbauer, and P. C. Hedgecock (1976), Plasma and magnetic field characteristics of the distant polar cusp near local noon: The entry layer, J. Geophys. Res., 81, 2883-2899, doi:10.1029/JA081i016p02883.

Shelley, E. G., R. D. Sharp, and R. G. Johnson (1976), $\mathrm{He}^{++}$and $\mathrm{H}^{+}$flux measurements in the day side cusp: Estimates of convection electric field, J. Geophys. Res., 81, 2363-2370, doi:10.1029/JA081i013p02363.

Topliss, S. M., C. J. Owen, and W. K. Peterson (2000), A simple model of complex cusp ion dispersions during intervals of northward interplanetary magnetic field, Geophys. Res. Lett., 27, 3587-3590, doi:10.1029/ 2000GL000123.

Trattner, K. J., S. A. Fuselier, W. K. Peterson, and C. W. Carlson (2002a), Spatial features observed in the cusp under steady solar wind conditions, J. Geophys. Res., 107(A10), 1288, doi:10.1029/2001JA000262.

Trattner, K. J., S. A. Fuselier, W. K. Peterson, M. Boehm, D. Klumpar, C. W. Carlson, and T. K. Yeoman (2002b), Temporal versus spatial interpretation of cusp ion structures observed by two spacecraft, J. Geophys. Res., 107(A10), 1287, doi:10.1029/2001JA000181.

Trattner, K. J., S. A. Fuselier, S. M. Petrinec, T. K. Yeoman, C. Mouikis, H. Kucharek, and H. Rème (2005), Reconnection sites of spatial cusp structures, J. Geophys. Res., 110, A04207, doi:10.1029/2004JA010722.

Trattner, K. J., J. S. Mulcock, S. M. Petrinec, and S. A. Fuselier (2007), Probing the boundary between anti-parallel and component reconnection during southwards IMF conditions, J. Geophys. Res., 112, A08210, doi:10.1029/2007JA012270.

Trattner, K. J., S. A. Fuselier, S. M. Petrinec, T. K. Yeoman, C. P. Escoubet, and H. Rème (2008), The reconnection site of temporal cusp structures, J. Geophys. Res., 113, A07S14, doi:10.1029/2007JA012776.

Tsyganenko, N. A. (1995), Modeling the Earth's magnetospheric magnetic field confined within a realistic magnetopause, J. Geophys. Res., 100, 5599-5612, doi:10.1029/94JA03193.

Wurz, P. (2000), Detection of energetic neutral particles, in The Outer Heliosphere: Beyond the Planets, edited by K. Scherer, H. Fichtner, and E. Marsch, pp. 251-288, Copernicus Gesellschaft e.V., Katlenburg-Lindau, Germany.

M. A. Dayeh and D. J. McComas, Southwest Research Institute, San Antonio, TX 78228, USA.

H. O. Funsten, Los Alamos National Laboratory, Los Alamos, NM 87545, USA.

S. A. Fuselier, S. M. Petrinec, and K. J. Trattner, Lockheed Martin Advanced Technology Center, Palo Alto, CA 94304, USA. (petrinec@ spasci.com)

D. Heirtzler, H. Kucharek, and E. Möbius, Institute for the Study of Earth, Oceans and Space, University of New Hampshire, Durham, NH 03824, USA.

P. Janzen and D. B. Reisenfeld, Department of Physics and Astronomy, University of Montana, Billings, MT 59101, USA.

T. E. Moore, Heliophysics Science Division, NASA Goddard Space Flight Center, Greenbelt, MD 20771, USA

N. A. Schwadron, Astronomy Department, Boston University, Boston, MA 02215, USA.

P. Wurz, Physics Institute, University of Bern, CH-3012 Bern, Switzerland. 